\title{
Artificial-intelligence Assisted, Label-free Imaging of Sub-cellular Biology
}

\author{
Arturo Burguete-Lopez ${ }^{1}$, Maksim Makarenko ${ }^{1}$, Fedor Getman ${ }^{1}$, Andrea \\ Fratalocchi $^{\dagger 1}$ \\ ${ }^{1}$ PRIMALIGHT, Faculty of Electrical Engineering; Applied Mathematics and Computational Science, King \\ Abdullah University of Science and Technology, Thuwal 23955-6900, Saudi Arabia \\ $\dagger$ Contact author: andrea.fratalocchi@kaust.edu.sa
}

\begin{abstract}
We discuss a technique enabling the recovery of refractive index and thickness maps of cells using bright-field microscopy and a suitably designed ultra-dark membrane. We show spatial resolutions as low as $50 \mathrm{~nm}$, enabling organelle differentiation. (c) 2021 The Author(s)
\end{abstract}

\begin{abstract}
Main Text
Due to their transparent nature, the lack of spectral signatures in the visible is a major challenge in the imaging of sub-cellular elements in biological microscopy $[1,2]$. Solving this problem has been the subject of considerable research effort for the past 70 years [3], with recent methods showing the possibility of extracting visible range signals of cellular components [4-6]. A key biophysical property of a cell is its refractive index. It can be correlated with the cell's mass, protein concentration and metabolic processes [7,8]. Furthermore, high resolution mapping of the refractive index enables the study of cell cycles, growing mechanisms and other processes with applications in the diagnosis and prevention of disease $[9,10]$.

There currently exist several proposed methods to optically recover refractive index maps of biological cells. Phase microscopy and digital holography enable refractive index measurements with sensitivities ranging from $10^{-2}$ to $10^{-4}$ RIU $[11,12]$. These techniques rely on measuring the optical path length (OPL) of the cell. As the OPL results from the product of the refractive index and thickness at each point, assumptions must be made on the thickness map of the cell, multiple images at different angles are required or additional equipment must be used to recover the thickness information [13].

In this work, we address these issues and present a technique capable of precisely recovering the thickness and refractive index maps of a cell with a single measurement. Our method exploits the appearance of structural colors emitted from sub-cellular features when the analyte is placed on top of a suitably engineered dark substrate, which stretches the cell and mitigates reflections, thus increasing the signal to noise ratio. We then use a conventional color camera to image the colors, with each camera pixel mapping the reflection spectra at a point of the cell to a triplet of RGB values in the resulting image. Each of RGB triplets contains point-wise, vectorial information on the thickness and refractive index of the cell, which is recovered using artificial intelligence techniques.

The substrate used for the proposed method is engineered by an industry-ready, scalable and low cost electroplating process, while any color (CMOS or CCD) camera can be used to characterize the samples. Experimentally, we achieved $50 \mathrm{~nm}$ resolution on control samples, which is sufficient to differentiate between the organelles of a cell in the refractive index maps.
\end{abstract}

\section{References}

1. S. A. Maier, Plasmonics: Fundamentals and Applications (Springer, London, 2007).

2. D. Punj, M. Mivelle, S. B. Moparthi, T. S. Van Zanten, H. Rigneault, N. F. Van Hulst, M. F. García-Parajó, and J. Wenger, "A plasmonic 'antenna-in-box'platform for enhanced single-molecule analysis at micromolar concentrations," Nat. nanotechnology 8, 512 (2013).

3. R. Barer, K. F. A. Ross, and S. Tkacyk, "Refractometry of living cells," Nature 171, 720-724 (1953).

4. M. Seydack, "Nanoparticle labels in immunosensing using optical detection methods," Biosens. bioelectronics 20 , 2454-2469 (2005).

5. L. Polavarapu, J. Pérez-Juste, Q.-H. Xu, and L. M. Liz-Marzán, "Optical sensing of biological, chemical and ionic species through aggregation of plasmonic nanoparticles,” J. Mater. Chem. C 2, 7460-7476 (2014).

6. P. C. Ray, "Size and shape dependent second order nonlinear optical properties of nanomaterials and their application in biological and chemical sensing," Chem. reviews 110, 5332-5365 (2010).

7. G. Bao and S. Suresh, "Cell and molecular mechanics of biological materials," Nat. Mater. 2, 715-725 (2003). 


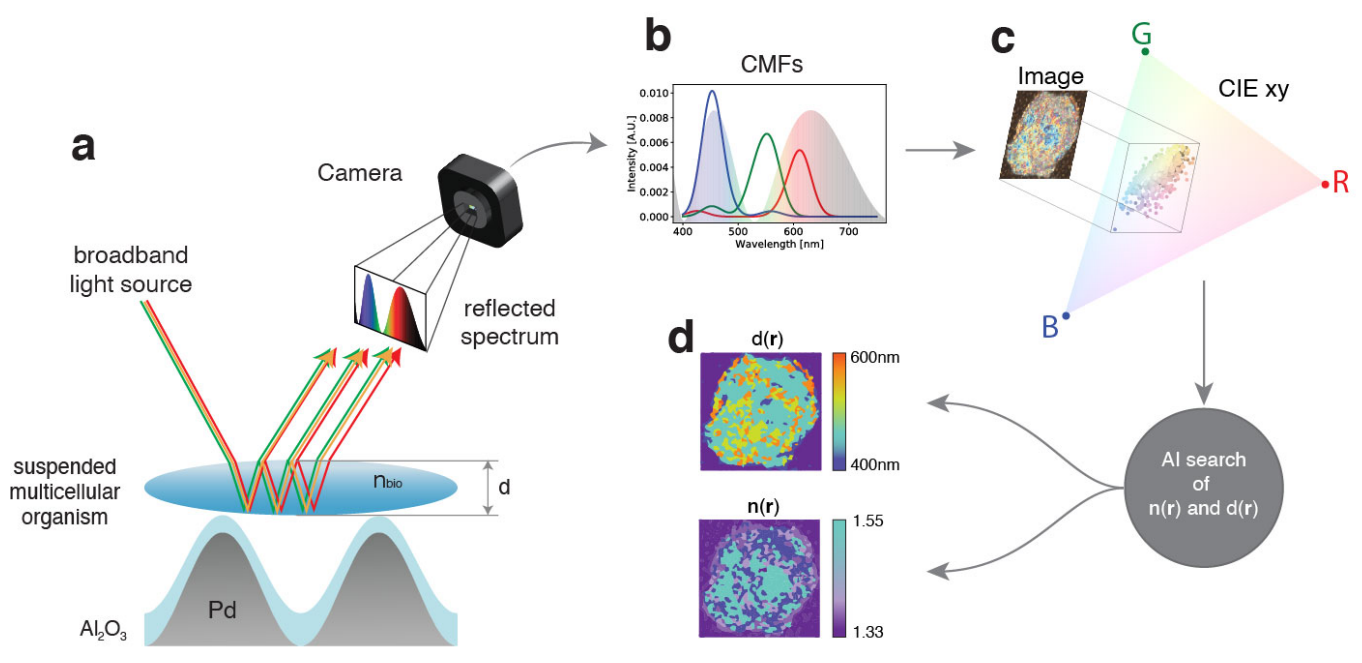

Fig. 1. (a) A cell placed on the dark substrate stretches and exhibits structural colors when illuminated with broadband light. The reflection spectra at each point of the cell is captured by a camera and reduced through the device's color matching functions (CMFs) (b) to a triplet of RGB values for producing a color image (c). The RGB values of each pixel in the image are mapped to the cell's local thickness and refractive index through an artificial intelligence algorithm, which allows recovering 2D maps of these quantities (d).

8. E. S. Boyden, F. Zhang, E. Bamberg, G. Nagel, and K. Deisseroth, "Millisecond-timescale, genetically targeted optical control of neural activity," Nat. Neurosci. 8, 1263-1268 (2005).

9. Y. Park, M. Diez-Silva, G. Popescu, G. Lykotrafitis, W. Choi, M. S. Feld, and S. Suresh, "Refractive index maps and membrane dynamics of human red blood cells parasitized by plasmodium falciparum," Proc. Natl. Acad. Sci. 105, 13730-13735 (2008).

10. M. Mir, Z. Wang, Z. Shen, M. Bednarz, R. Bashir, I. Golding, S. G. Prasanth, and G. Popescu, "Optical measurement of cycle-dependent cell growth," Proc. Natl. Acad. Sci. 108, 13124-13129 (2011).

11. K. G. Phillips, S. L. Jacques, and O. J. T. McCarty, "Measurement of single cell refractive index, dry mass, volume, and density using a transillumination microscope,” Phys. Rev. Lett. 109, 118105 (2012).

12. W. Choi, C. Fang-Yen, K. Badizadegan, S. Oh, N. Lue, R. R. Dasari, and M. S. Feld, "Tomographic phase microscopy," Nat. Methods 4, 717-719 (2007).

13. P. Y. Liu, L. K. Chin, W. Ser, H. F. Chen, C.-M. Hsieh, C.-H. Lee, K.-B. Sung, T. C. Ayi, P. H. Yap, B. Liedberg, K. Wang, T. Bourouina, and Y. Leprince-Wang, "Cell refractive index for cell biology and disease diagnosis: past, present and future," Lab Chip 16, 634-644 (2016). 